\title{
Line Scratches Dètection and Restoration via Light Diffraction
}

\author{
V. Bruni, D. Vitulano \\ Istituto per le Applicazioni \\ del Calcolo "M. Picone", C. N. R. \\ Viale del Policlinico 137 \\ 00161 Rome, Italy \\ Tel. +39--6-88470224, Fax +39-6-4404306 \\ \{bruni,vitulano\}@iac.rm.cnr.it
}

\author{
A. Kokaram \\ Electronic and Electrical \\ Engineering Department \\ Trinity College, College Green, \\ Dublin 2, Ireland \\ Tel. 3531608 3412, Fax: +35316772442 \\ anil.kokaram@tcd.ie
}

\begin{abstract}
This paper presents a unified model for the detection and removal of line scratches. It is based on modelling the scratch effect by allowing for the diffraction of light. The paper gives some evidence as to why light diffraction can give rise to scratches. The physical modelling of the defect along with its classification as region of partially missing data allows very good results both in detection and in restoration.
\end{abstract}

Keywords: Digital Film Restoration, Scratch Detection and Restoration, Light Diffraction.

\section{Introduction}

Scratches are common defects on old film sequences. They appear as straight lines subtending much of the vertical extent of an image frame. The angle to the vertical is up to $5 \%$ [8]), with bright or dark intensity and a width from 3 to 10 pixels [6]. Since they occupy the same or quite the same location in subsequent frames, they cannot be classified as temporally impulsive defects. Moreover their automatic detection is usually harder than their recovery because there is some confusion as to what separates a line scratch defect from any other thin line in an image. For that reason there are more proposals for restoration than for detection. In order to propose an effective and unified model, some empirical observations, often exploited by experts, should be accounted for.

Color The transport mechanism can impinge either on the side of the support material (negative side) or on the other side (positive side). This leads to black and white scratches respectively. It means that for white scratches a part of the information is definitively obliterated, since the mechanism "throws away information". On the contrary, with regard to dark scratches no information loss is incurred since it is only the support material that is damaged. Therefore, for dark scratches

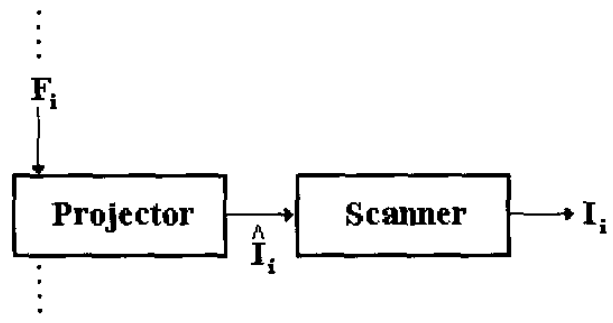

Figure 1. Scheme of digitalization process of a frame $F_{i}$.

a simple transformation between the image and the degraded copy should exist.

Scanning The images are acquired by a film scanner. The scanner uses CCD cells and these cause a spread of the light which illuminates them (see Fig. 1).

A simple scheme of the digitization process is depicted in Fig. 1 where $F_{i}$ is the degraded frame, $\hat{I}_{i}$ is the projected image (where the scratch emerges as a diffraction effect) and $I_{i}$ is the real observed image (incorporating the light spreading effect of the CCD array).

Using these observations, the novel contribution in this paper is to prove that scratches on digital images are light diffraction effects (see Appendix A) and then to propose a unified model for both detection and restoration based on this effect. The scratch is therefore constrained to follow a $\sin c^{2}$ behaviour as with light diffraction. Its cross-section [6] can be then defined as follows:

$$
L_{n}(i)=b_{s} \operatorname{sinc} c^{2}\left(\omega\left|i-c_{p}\right|\right)
$$

where $b_{s}$ is the brightness of the scratch, $c_{p}$ its location while $\omega$ is a parameter to be estimated.

The paper is organized as follows. In Section 2 a model for line scratches detection is proposed and some experimental results are shown. A model for restoration, its implementation and some experimental results are the topics 
of Section 3. Finally Section 4 draws the conclusions, while in Appendix A diffraction for line scratches is proved.

\section{Detection}

As previously mentioned, one of the main difficulties in the automatic detection of scratches is their occurrence in the same location in subsequent frames. In [5] this temporal continuity is exploited by tracking the detected artifact lines over the image sequence by means of a Kalman filter. But a precise detection on the first frame is required. On the contrary, in [6] an additive spatial model is proposed. Scratches are detected by means of a Bayesian refinement strategy starting from an initial guess obtained by a simple median filter based line detection process. Both the previous techniques fail to detect a subset of scratches, particularly the low contrast and shortest ones. Moreover, they are somewhat sensitive to thresholds.

A model for detection (SDHO) based on the Weber's law has been proposed in [3]. Its peculiarity was the representation of a scratch as a region of partially missing data, i.e. the model was not completely additive. In fact, empirical observations proved that additivity of image information and scratch in the cross-section depends on the visibility of the scratch itself - that is its brightness compared to the image surround. Moreover, scratch information vanishes moving away from its maximum. Thus the model can be written as follows

$$
\begin{aligned}
& I(i)=\left(1-(1-\gamma) k_{p}^{\left|i-c_{p}\right|}\right) \quad G^{\prime}(i)+ \\
& +(1-\gamma) \quad k_{p}^{\left|i-c_{p}\right|} L_{n}^{(p)^{\prime}}(i)
\end{aligned}
$$

where $I$ is the degraded image brightness, $G^{\prime}$ is the original image one, $\gamma$ is a normalized coefficient representing the ratio between the brightness of the scratch and the image one while $k_{p}$ is the decay of the line profile. $L_{n}^{(p)^{\prime}}$ is the scratch model. The aim was to model the scratch profile with a damped harmonic oscillator. Only two of the three solutions were employed: critical and under damping, i.e.

$$
L_{n}^{(p)^{\prime}}(i)= \begin{cases}b_{p}\left(-\frac{2\left|i-c_{p}\right|}{m}+1\right) & \text { critical damping } \\ b_{p} \cos \left(\frac{\pi\left|i-c_{p}\right|}{m}\right) & \text { underdamping }\end{cases}
$$

and

$$
k_{p}= \begin{cases}e^{-2 / m} & \text { critical damping } \\ e^{-1 / 2 m} & \text { underdamping. }\end{cases}
$$

\subsection{A new contribution}

Appendix A illustrates that the scratch behaviour arises from light diffraction. Therefore eq. (1) is more appropriate for use in (2). Estimating the free parameters in (1) is then the main issue. In (3) the frequency of the cosine is $\pi / m$ : at a distance equal to $m$ from zero there is the first relative extremum. Using $\omega=\frac{\alpha}{m}$ yields

$$
L_{n}(x)=b_{s} \frac{\sin ^{2}(\alpha \pi x / m)}{(\alpha \pi x / m)^{2}}, \quad x=\left|i-c_{p}\right|
$$

\begin{tabular}{|l|r|r|r|}
\hline & SITDOWN & KNIGHT & STAR \\
\hline True scratches & 24 & 1 & 21 \\
\hline Kokaram's model & 9 & 1 & 1 \\
\hline Damped sinusoid & 12 & 1 & 11 \\
\hline SDHO & 19 & $-\frac{1}{1}$ & 18 \\
\hline Diffraction & 18 & $\frac{1}{1}$ & 12 \\
\hline
\end{tabular}

Table 1. Comparison between Kokaram algorithm [6] results, damped sinusoid based one [9], SDHO (harmonic oscillator) [3] and the proposed one (diffraction).

Local extrema (minima if $b_{s}>0$ ) are located where the function $\sin ^{2}$ is zero, i.e. $x=\frac{m k}{\alpha}$. Since the first minimum is $x=m$, it is trivial to see that $\alpha=1$ and then

$$
L_{n}(x)=b_{s} \sin c^{2}(x / m)
$$

Since image luminance cross-section is a zero-mean function, while $\sin c^{2}$ is a function everywhere positive, then $b_{s}=b_{p}-a$, where $b_{p}$ and $a$ are the brightness values of the image cross-section respectively at $c_{p}$ and its closest extremum location (if there is perfect symmetry). Then, in order to make $\operatorname{sinc}^{2}$ to be zero-mean, a shift of $L_{n}(x)$ along $\mathrm{y}$-axis is required as follows.

$$
L_{n}(x)=\left(b_{p}-a\right) \operatorname{sinc}^{2}(x / m)+a .
$$

Notice that the maxima of the function $\operatorname{sinc} c^{2}$ has an amplitude (with respect to the main maximum) equal to $4.7 \%$, $1.6 \%, .8 \%, \ldots$ located near $(k+1 / 2) \pi, \quad k \in N$. The behaviour of the damping of maxima amplitudes can be modelled by means of an exponential function $f(x)$ (exactly at least for the first secondary maximum). Such a function will depend on $m$ and it is easy to show that it can be described by the following equation:

$$
f(x)=e^{\frac{-2 x}{m}} .
$$

Then the model equation ( 2 ) becomes:

$$
I(i)=\left(1-(1-\gamma) e^{\left.\frac{-2}{m} \mid i-c_{p}\right\}}\right) G^{\prime}(i)+(1-\gamma) L_{n}(i)
$$

where $L_{n}(i)=L_{n}\left(\left|i-c_{p}\right|\right)=L_{n}(x)$.

The following are of importance.

- The damping of $\sin c^{2}$ has the same behaviour as the critical damping of the harmonic oscillator.

- Since $\sin c^{2}$ is a non-negative function, the parameter $a$ has to be estimated from the data and it is equal to the average of the data in the range $\left[c_{p}-m, c_{p}+m\right]$.

\subsection{Experimental Results}

We have performed our detection model on three frames of three classical sequences [6]: Star (1-st) (Fig. 2), Knight 


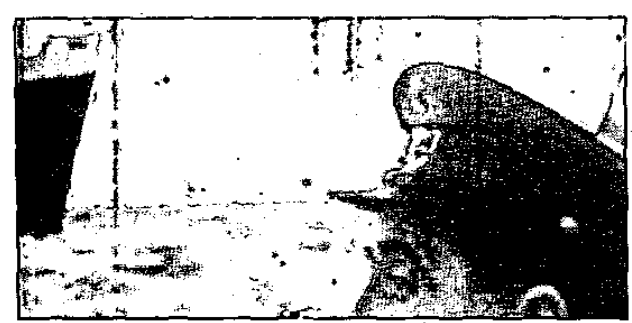

Figure 2. $1^{\text {st }}$ frame of the degraded Star sequence

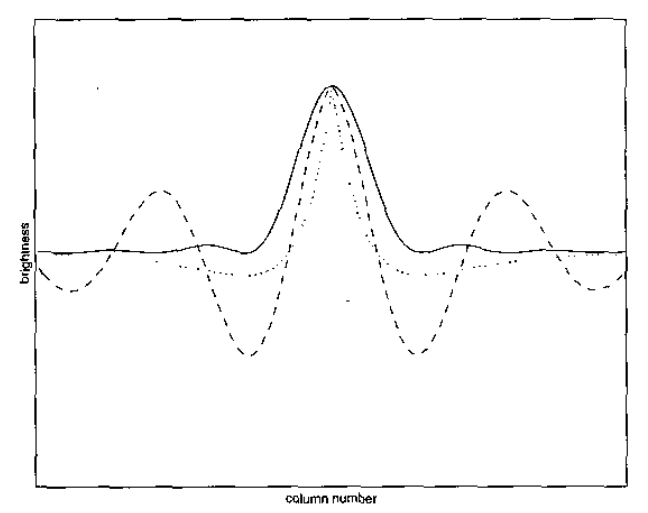

Figure 3. $\operatorname{sinc}^{2}$ (solid), critical damping (dotted) and underdamping (dashed) profiles.

(33-rd) (Fig. 5) and Sitdown (7-rd frame) (Fig. 7). In Table 1 there are some results we achieved and they are compared with existing models. Results indicate that the empirical model (SDHO) is better than the real physical one. The empirical model is more general since it considers the embedded correlated noise due to the original image. In other words it is less restrictive for the scratch profile model and in the meantime it includes the "pure" diffraction case (i.e. without correlated information due to the image) (See Fig. 3).

Unlike detection, the restoration phase requires a precise knowledge of scratch profile. To do this, diffraction has to be exploited since it gives the true information of the scratch to be removed yielding a precise recovery, as we will see in the next Section.

\section{Restoration}

Previous approaches for restoration $[1,2,4,5,6,7]$, are based on the assumption that regions affected by scratches do not contain information. The work of [1] and [4], which introduces the concept of inpainting, can also be applied to old movies. But in this case, the hypothesis "there is no significant information in the region to be inpainted" (see [1] page 1 section 2 rows 5-6) is not true. Moreover, variational

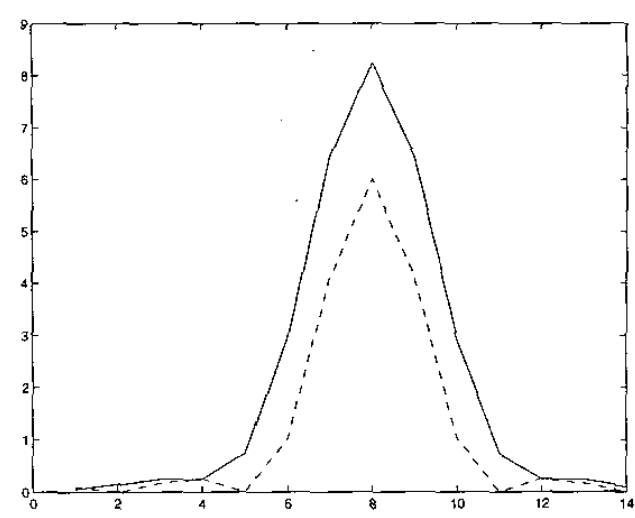

Figure 4. $\sin c^{2}$ (dashed), convolution of $\sin c^{2}$ with a gaussian filter with $\sigma=3$ (solid)

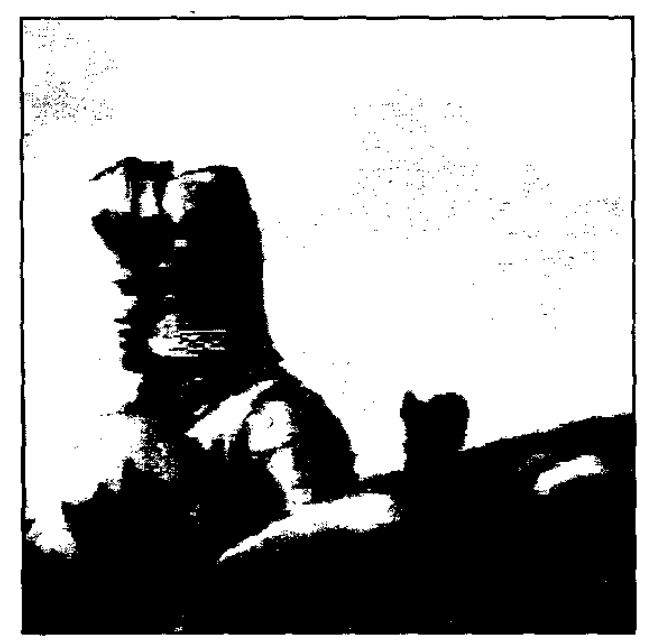

Figure 5. $33^{\text {th }}$ frame of the degraded Knight sequence.

approaches, though applied in small regions of the image, are expensive. In [2], [4], [5], and [7], again, information in the degraded regions is recovered as "extension" of the closest surround. Finally it is worth emphasizing that in [2], for the first time a diffraction hypothesis has been made even though it has not been proved or exploited.

It transpires that scratches sometimes remove a part, but not the whole information from the original image. The strength (brightness) of the diffraction effect is due to the depth of the scratch on the film material. Incident light must pass through the remaining amount of material and the cross section of the cut in the material may lead to an asymmetry in the scratch. In the detection phase we assumed the scratch symmetric. But this was a first approximation since we was only interested in achieving a threshold value and then a basis for decision. Moreover it was good enough since the cross section represents the sum of various asym- 


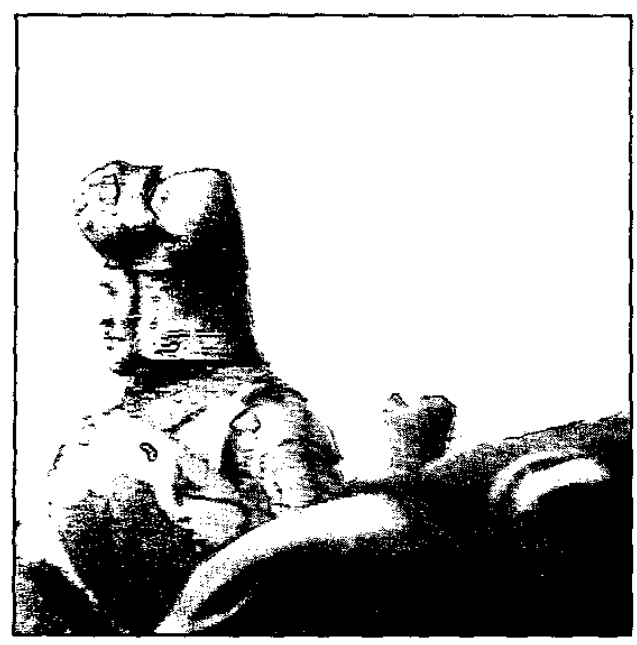

Figure 6. Restored $33^{\text {th }}$ frame of Knight sequence.

metric rows. A good restoration entails a good approximation of the scratch and that implies that asymmetry has to be estimated.

From the previous considerations and from the detection step, we know that the scratch information (i.e the crosssection) on the degraded image follows a $\sin c^{2}$ function. We know where it is located $\left(c_{p}\right)$ and its width $(2 m)$ in a suitable region $\Omega$ of the image but the amplitude $\left(b_{p}\right)$ is unknown. However, from the cross-section we have a value of $b_{p}$, but this is affected by the "original image noise". A least squares estimation process follows.

Starting from the equation of the scratch profile on the image cross-section

$$
L_{n}(i)=b_{p} \operatorname{sinc} c^{2}\left(\frac{\left|i-c_{p}\right|}{m}\right), \quad c_{p}-m \leq i \leq c_{p}+m,
$$

$b_{p}$ value can be estimated from the data in the least square sense, i.e minimizing the mean square error in the scratch domain $D=\left[c_{p}-m, c_{p}+m\right]$. It is a simple linear problem because (9) can be written as follows $L_{n}(i)=b_{p} F(i)$ where the function $F(i)=\operatorname{sinc}^{2}\left(\frac{\left|i-c_{p}\right|}{m}\right)$ is known when the domain $D$ is fixed. Then, if $I(i)$ are the corrupted data of the image signal (cross-section of $\Omega$ ), we have to find $b_{p}$ :

$$
b_{p}=\min _{\alpha \in \mathcal{R}} \sum_{i \in D}|I(i)-\alpha F(i)|^{2} .
$$

At this point, for a given region of the image $\Omega$, we have all parameters for the scratch (i.e. $b_{p}, c_{p}, m$ ). These can be used to restore each row belonging to $\Omega$. A filter $w$ to apply to the degraded data has to be designed. Considering the model equation (8) we define the filter coefficients (Wiener filter), as follows:

$$
w(i)=\frac{\left(I(i)-C_{2} S(i)\right)^{2}}{\left(I(i)-C_{2} S(i)\right)^{2}+\left(\frac{\bar{C}_{2}}{C_{1}} S(i)\right)^{2}}, \quad i \in D
$$

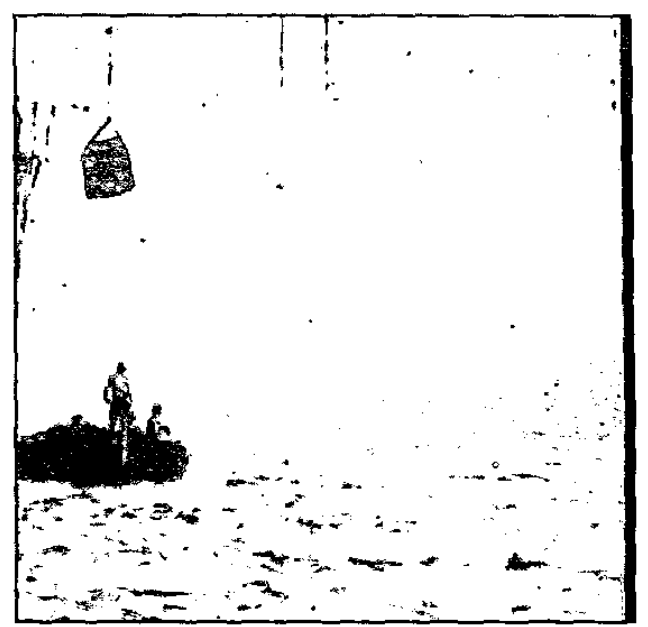

Figure 7. $7^{\text {th }}$ frame of the degraded Sitdown sequence.

where $C_{1}=\left(1-(1-\gamma) e^{\frac{-2}{m}\left|i-c_{p}\right|}\right), C_{2}=(1-\gamma)$ and $S(i)=C L_{n}(i)$ (C is a constant depending on the features of the scanner and it will be defined later). Hence each row which contributes to $\Omega$ cross-section is processed with the filter $w$. It is obvious that the smaller $\Omega$, the more precise the attenuation (the data are quite similar).

\subsection{The Algorithm}

- For each region $\Omega$ of the image containing only one scratch, the amplitude $b_{p}$ of the $\sin c^{2}$ is estimated from its cross-section in $D$ in the least square sense (as in $(10)$ );

- for each row $r$ of $\Omega$

1. The cross-section $I_{r}(i)$ is computed, i.e. we subtract its local mean $M_{r}(i)$;

2. The filter domain $D_{r}$ is computed. The maximum of $I_{r}(i)$ in $D$ is found, and its adjacent left and right minima. If $c_{p}^{(r)}, c_{p}^{(r)}-m_{s}$ and $c_{p}^{(r)}+m_{d}$ are respectively the locations of the maximum, the left minimum and the right minimum, then $D_{r}=\left[c_{p}^{(r)}-m_{s}, c_{p}^{(r)}+m_{d}\right]$;

3. The $\sin c^{2}$ is adapted to the new local scratch parameters, i.e.

$L_{n}^{(r)}(i)=$

$$
= \begin{cases}b_{p} \sin c^{2}\left(\frac{\left|i-c_{p}^{r}\right|}{m_{s} \mid}\right), & i \in\left[c_{p}^{(r)}-m_{s}, c_{p}^{(r)}\right] \\ b_{p} \sin c^{2}\left(\frac{\left|i-c_{p}^{r}\right|}{m_{d}}\right), & i \in\left(c_{p}^{(r)}, c_{p}^{(r)}+m_{d}\right]\end{cases}
$$

4. Filter coefficients are defined as follows.

$$
\begin{aligned}
w_{r}(i) & =\frac{\left(I_{r}(i)-C_{2} S_{r}(i)\right)^{2}}{\left(I_{r}(i)-C_{2} S_{r}(i)\right)^{2}+\left(\frac{C_{2}}{C_{1}} S_{r}(i)\right)^{2}}, \\
\text { with } \quad i & \in D_{r} \text { and where } S_{r}(i)=C L_{n}^{(r)}(i) ;
\end{aligned}
$$




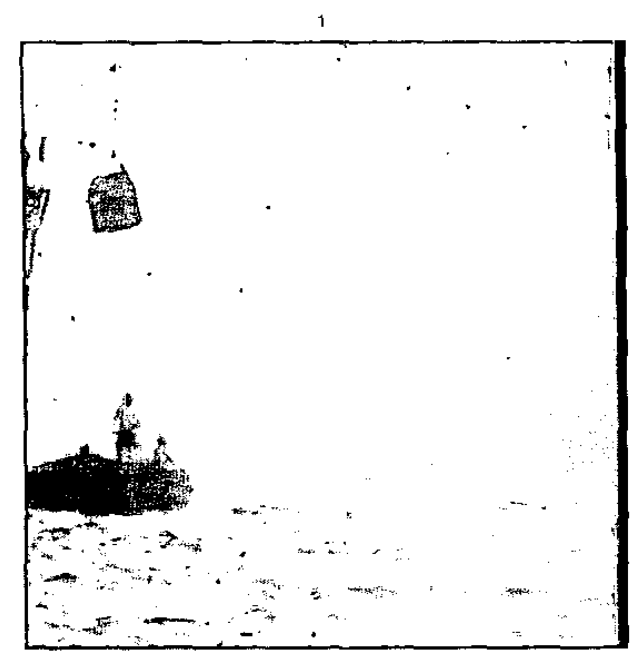

Figure 8. Restored $7^{\text {th }}$ frame of Sitdown sequence.

5. This filter is applied to the row cross-section $I_{r}$ obtaining the denoised cross-section $\hat{I}_{r}(i)=$ $w_{r}(i) I_{r}(i), \quad i \in D_{r}$

6. The local mean of $L_{n}\left(\sin c^{2}\right.$, i.e. scratch information) is computed and subtracted from the local mean $M_{r}(i)$ of the selected row, obtaining the denoised local mean of the row $\hat{M}_{r}(i)$;

7. The restored row is given by $\hat{M}_{r}(i)+\hat{I}_{r}(i)$.

Step no. 2 computes the asymmetry. It is necessary because the scratch domain $D$ is estimated from the crosssection of the whole region $\Omega$ and then it gives a global information of the scratch in this region. 'A symmetric crosssection can come from several asymmetric rows. On the contrary, at this stage we need the precise local features of the scratch in order to completely remove it. Since we are investigating the local parameters of the scratch, the hypothesis of symmetry of the defect are not necessarily verified. For that reason we estimate $m_{s}$ and $m_{d}$ and then we define two different $\sin c^{2}$ on the left and on the right of the scratch location. Moreover asymmetry comes from the fact that transport mechanism causes not perfect slits. Then scratch is the result of a more complicated effect.

Step no. 6 is supported by the fact that $M_{r}(i)$ are the local mean of the degraded row and then they also contain scratch information. Therefore, in order to achieve a good restoration, this information has to be subtracted from them. The scratch contribution in $M_{r}(i)$ is represented by the local mean of the $\operatorname{sinc} c^{2}$ in the selected region. Splitting information in low frequency and high frequency components has already been exploited in [2] and [5].

\subsection{Some considerations and experimental results}

Before showing the experimental results we highlight two interesting aspects concerning the scanning device. The parameter $\gamma$, estimated in the detection step, represents a measure of the visibility of the scratch on the whole image according to the Weber's law. (Keep in mind that $\gamma=\frac{E_{m}}{\alpha b_{p}}$, where $E_{m}$ is the average of the peaks amplitude of the cross-section and $\alpha$ is a normalization factor [9]). The visibility of a scratch with brightness $b_{2}$ with respect to another scratch $b_{1}$ can be measured finding the parameter $\beta: \beta \gamma_{1}=\gamma_{2}$; hence $\beta=\frac{b_{2}}{b_{1}}$. For that reason the constant $C$ has been introduced in (11) as follows:

$$
C=p_{\sigma} \frac{b_{i}}{b_{1}}
$$

where $b_{i}$ is the amplitude of the scratch we are removing, $b_{1}$ is the amplitude of a fixed scratch and $p_{\sigma}$ is a parameter depending on the scanner features. This procedure is required to overcome the lack of knowledge of the scanner features.

The scanner also contributes to the shape of the scratch. In fact its profile does not exactly coincide with a $\sin c^{2}$ behaviour. We assume that scratch profile comes from the convolution of a $\sin c^{2}$ with a zero mean gaussian function with variance $\sigma^{2}$. From some experimental observation, we noticed that, if $\sigma$ is in a suitable range, the result is an amplified and dilated $\operatorname{sinc}^{2}$ (see Fig. 4). This contribution is contained in the parameter $p_{\sigma}$.

The proposed model has been tested on many frames. In Fig. 6 and in Fig. 8 are shown results achieved on Knight (33rd frame) and Sitdown ( 7 th) sequences. It can be noticed that even though surround information has not been used, recovery achieves good results in terms of visual quality.

\section{Conclusions}

In this paper we have presented a model exploiting the diffraction effect for both detection and restoration of line scratches. While black scratches are quite exactly described by the effect above, the white ones are not. For these latter scratches, the transport mechanism acts on the positive side of the film material. Hence, only in this case part of the information is definitively obliterated as much as the depth of the scratch. This fact has also been confirmed by experiments. Hence, our future research will focus on an improvement of the proposed model integrating it with autoregressive filters based ones, as in [6].

\section{Acknowledgements}

Authors would like to thank RTP, Lisbon, Portugal for making available test sequences used in this paper and Cinecittá Studios experts for very interesting discussions and suggestions. 


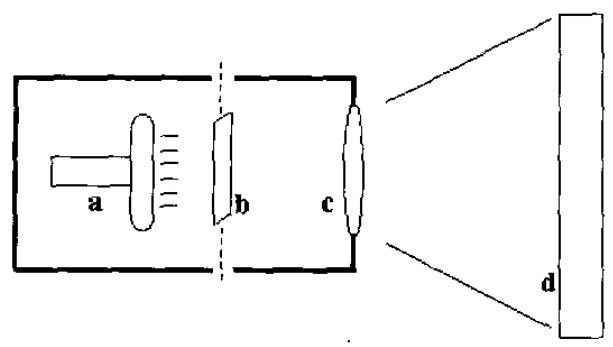

Figure 9. Scheme of a fixed focal lens based projector: $a$ is the lamp, $b$ is the film, $c$ is the lens and $d$ is the screen.

\section{A. Diffraction}

We have diffraction when:

1. $\lambda<<d$, where $\lambda$ is the wavelength of the light which passes through a slit and $d$ is the width of the slit; and

2. $D \simeq \infty$, where $D$ is the distance between the slit and the screen( $\equiv$ big enough).

We show here that diffraction conditions for scratches in old movies exist in film projector as well as in film scanning devices.

Usually a projection device for $35 \mathrm{~mm}$ film is composed of a light source (lamp) illuminating the film to project the image on a lens whose only purpose is to increase the size of the projected image on the screen (see Fig. 9). Features of the lens are designed in agreement with the size of both the projection room (features: focal distance and conjugate points) and the screen (feature: magnification ratio). Firstly, light ray directions are parallel in the projector. This is in agreement with a precise request for a diffraction experiment.

If we indicate the wavelength of the light rays of the lamp with $\lambda$, we know that $l_{1} \leq d_{p} \leq l_{2}$ (see Introduction) where $d_{p}$ is the scratch width on a digital image. Then it is easy to see that

$$
\frac{l_{1} \cdot G_{r}}{G_{p}} \leq d_{s} \leq \frac{l_{2} \cdot G_{r}}{G_{p}}
$$

where $G_{r}$ and $G_{p}$ respectively indicate the (original analogical) image size (in $\mathrm{cm}$ ) and the digital image size (in pixel).

At this point, we exploit the well-know diffraction relation:

$$
d=\frac{2 D \lambda}{d_{s}}
$$

where $d$ is the scratch's width on the film material, $D$ is the distance between the slit and the screen (in our case the lens) while $\lambda$ and $d_{s}$ have previously been defined. Combining (12) and (13) it is easy to obtain the first diffraction condition:

$$
d_{\min } \simeq \frac{2 D \lambda_{\min } G_{p}}{l_{2} G_{r}} \simeq 210^{2} \lambda_{\min }>>\lambda_{\max }
$$

considering $.39 \leq \lambda \leq .78 \mu \mathrm{m}, l_{1}=3$ and $l_{2}=10, G_{p}$ at PAL resolution and $G_{r}$ without image size increasing $(\simeq$ $35 \mathrm{~mm}), D \simeq 5 \mathrm{~cm}$.

The situation we have described above is relative to old projectors that had fixed focal distance (in the modern ones this distance may be adaptively changed). Note that this simplified situation is not restrictive at all, since a modern projector behaves as an old one once the focal length of the lens is fixed.

Moreover it is easy to show that the second constraint for the diffraction effect is verified, i.e. $D$ much bigger than both $\lambda$ and $d$ (there are some size orders of difference). Note that in this academical example we don't know in advance the focal distance of the lens since tied to the size of the room for the movie projection and the projection screen. As matter of fact, we are not interested in this information, but we have to keep in mind that when $D$ approaches $\lambda$ the diffraction effect is less and less evident and the light is almost completely in the centre of the screen. Finally, it is also easy to verify that when a modern film scanner (or telecine machine) is involved, diffraction already exists. In fact, in Fig. 9 CCD cells lie on $c$ and $D$ is shorter. Then in (13) both $D$ and $d_{s}$ decrease (about one size order) leaving the ratio $\frac{D}{d_{s}}$ mainly unchanged.

\section{References}

[1] M. Bertalmio, G. Sapiro, V. Caselles, and C. Ballester. Image inpainting. Computer Graphics, SIGGRAPH 2000, July 2000.

[2] T. Bretschneider, O. Kao, and P. Bones. Removal of vertical scratches in digitised historical film sequences using wavelet decomposition. Proceedings of Image and Vision Computing New Zealand, pages 38-43, 2000

[3] V. Bruni and D. Vitulano. Scratch detection via underdamped harmonic motion. Proceedings of IEEE Intermational Confer. ence ICPR 2002, Quebec City, Canada, August 2002.

[4] S. Esedoglu and J. Sheno. Digital inpainting based on the mumford-shah-euler image model. European J. Appl. Math, 13:353-370, 2002.

[5] L. Joyeux, S. Boukir, and B. Besserer. Film line removal using kalman filtering and bayesian restoration. WACV'2000, IEEE Workshop on the Application of Computer Vision, Palm Springs, California, December 2000.

[6] A. Kokaram. Motion Picture Restoration: Digital Algorithms for Artefact Suppression in Degraded Motion Picture Film and Video. Springer-Verlag, 1998.

[7] L. Maddalena. Efficient methods for scratch removal in image sequences. Proceedings of IClAP 200I, $11^{\text {th }}$ Intemational Conference on Image Analysis and Processing, Palermo, Italy, September 2001.

[8] P. Schallauer, A. Pinz, and W. Haas. Automatic restoration algorithms for $35 \mathrm{~mm}$ film. VIDERE: Joumal of Computer Vision Research, The MIT Press, 1(3), 1999.

[9] D. Vitulano, V. Bruni, and P. Ciarlini. Line scratch detection on digital images: An energy based model. Special Issue of Journal of WSCG, 10(2):477-484, 2002. 